\title{
LIOUVILLIAN FIRST INTEGRALS FOR A CLASS OF GENERALIZED LIÉNARD POLYNOMIAL DIFFERENTIAL SYSTEMS
}

\author{
JAUME LLIBRE ${ }^{1}$ AND CLÀUDIA VALLS ${ }^{2}$
}

\begin{abstract}
We study the existence of Liouvillian first integrals for the generalized Liénardpolynomial differential systems of the form $x^{\prime}=y, y^{\prime}=-g(x)-f(x) y$, where $f(x)=3 Q(x) Q^{\prime}(x) P(x)+Q(x)^{2} P^{\prime}(x)$ and $g(x)=Q(x) Q^{\prime}(x)\left(Q(x)^{2} P(x)^{2}-\right.$ $1)$ with $P, Q \in \mathbb{C}[x]$. This class of generalized Liénard polynomial differential systems has the invariant algebraic curve $(y+Q(x) P(x))^{2}-Q(x)^{2}=0$ of hyperelliptic type.
\end{abstract}

\section{IntroduCtion AND STATEMENT OF THE MAIN RESUlt}

One of the more classical and difficult problem in the qualitative theory of planar differential systems depending on parameters is to characterize the existence and non-existence of first integrals in function of the parameters of the system.

We consider the polynomial differential system

$$
x^{\prime}=y, \quad y^{\prime}=-g(x)-f(x) y,
$$

called the generalized Liénard polynomial differential system, where $x$ and $y$ are complex variables and the prime denotes derivative with respect to the time $t$, which can be real or complex. Such differential systems appear in several branches of the sciences, such as biology, chemistry, mechanics, electronics, etc, see for instance $[6,17]$ and the references quoted there. For $g(x)=x$ the Liénard differential system (1) is called the classical Liénard polynomial differential system.

Let

$$
X=y \frac{\partial}{\partial x}-(g(x)+f(x) y) \frac{\partial}{\partial y}
$$

be the polynomial vector field associated to system (1). Let $U$ be an open and dense set in $\mathbb{C}^{2}$. We say that the non-locally constant function $H: U \rightarrow \mathbb{C}$ is a first integral of the polynomial vector field $X$ on $U$, if $H(x(t), y(t))=$ constant for all values of $t$ for which the solution $(x(t), y(t))$ of $X$ is defined on $U$. Clearly $H$ is a first integral of $X$ on $U$ if and only if $X H=0$ on $U$.

A Liouvillian first integral is a first integral $H$ which is a Liouvillian function, that is, roughly speaking which can be obtained "by quadratures" of elementary functions. For a precise definition see [16]. The study of the Liouvillian first integrals is a classical problem of the integrability theory of the differential equations which goes back to Liouville.

2010 Mathematics Subject Classification. 34C35, 34D30.

Key words and phrases. Darboux polynomial, invariant algebraic curve, exponential factor, Liouvillian first integral, Liénard polynomial differential system. 
As far as we know the Liouvillian first integrals of some multi-parameter family of planar polynomial differential systems has only been completely classified for the planar Lotka-Volterra systems, see $[1,7,12,13,14,15]$.

Note that when $g(x)=x$, system (1) is the well-known classical Lienard polynomial differential system whose Liouvillian first integrals were studied in [9]. Moreover, when $2 \leq \operatorname{deg} g \leq \operatorname{deg} f$ the Liouvillian first integrals of these systems were studied in [10], and when $\operatorname{deg} g=\operatorname{deg} f+1$ the Liouvillian first integrals of these systems were studied in [11].

The case of $f$ and $g$ being general polynomials is out of the reach right now. The study of Liouvillian first integrals is based on particular on the search of what is called an invariant algebraic curve. Let $h=h(x, y) \in \mathbb{C}[x, y] \backslash \mathbb{C}$. As usual $\mathbb{C}[x, y]$ denotes the ring of all complex polynomials in the variables $x$ and $y$. We say that $h=0$ is an invariant algebraic curve of the vector field $X$ if it satisfies

$$
y \frac{\partial h}{\partial x}-(g(x)+f(x) y) \frac{\partial h}{\partial y}=K h,
$$

for some polynomial $K=K(x, y) \in \mathbb{C}[x, y]$ called the cofactor of $h=0$. Clearly $h$ has degree at most $m=\max \{\operatorname{deg} f+1, \operatorname{deg} g\}-1$. We also say that $h$ is a Darboux polynomial of system (1). Note that a polynomial first integral is a Darboux polynomial with zero cofactor.

The invariant algebraic curves are important because a sufficient number of them forces the existence of a first integral. This result is the basis of the Darboux theory of integrability, see for instance $[4,5,8]$.

An exponential factor $E$ of system (3) is a function of the form $E=\exp (u / v) \notin \mathbb{C}$ with $u, v \in \mathbb{C}[x, y]$ satisfying that

$$
y \frac{\partial E}{\partial x}-(g(x)+f(x) y) \frac{\partial E}{\partial y}=L E,
$$

for some polynomial $L=L(x, y)$ of degree at most $m$, called the cofactor of $E$.

It is easy to check the following result for any generalized Liénard polynomial differential system (1).

Proposition 1. System (1) has the exponential factors $\exp \left(x^{j}\right)$ with cofactors $x^{j-1} y$ for $j=1, \ldots, \max \{\operatorname{deg} f, \operatorname{deg} g-1\}$ and the ones of the form $\exp (u(x))$ with $u(x) a$ polynomial of degree at most $\max \{\operatorname{deg} f, \operatorname{deg} g-1\}$. Moreover, if $\operatorname{deg} g \leq \operatorname{deg} f$ then system (1) has the exponential factors $\exp \left(x+\int f(x) d x\right)$ with cofactor $-g(x)$.

The main difficulty for studying the Liouvillian integrability of a polynomial differential system is the characterization of the invariant algebraic curves and also the characterization of the exponential factors of the polynomial differential system. For that reason we restrict our study of the Liovillian integrability of the generalized Liénard polynomial differential systems (1) to the following ones

$$
\begin{aligned}
x^{\prime} & =y, \\
y^{\prime} & =-g(x)-f(x) y \\
& =-Q(x) Q^{\prime}(x)\left(Q(x)^{2} P(x)^{2}-1\right)-\left(3 Q(x) Q^{\prime}(x) P(x)+Q(x)^{2} P^{\prime}(x)\right) y .
\end{aligned}
$$

Our main result on the Liouvillian integrability of the class of generalized Liénard polynomial differential system (3) is the following. 
Theorem 2. The following statements hold for the generalized Liénard polynomial differential system (3).

(a) When $\operatorname{deg} Q=0$, i.e., $Q(x)=\kappa \in \mathbb{C}$, then system (3) is Liouvillian integrable with the first integral $H=y+\kappa^{2} P(x)$;

(b) When $\operatorname{deg} P=0$, i.e., $P(x)=\kappa \in \mathbb{C}$, then system (3) is Liouvillian integrable with the first integral

$$
H=\frac{\kappa^{2} Q(x)^{2}+\kappa y-1}{\sqrt{y^{2}+(2 \kappa y-1) Q(x)^{2}+\kappa^{2} Q(x)^{4}}} .
$$

(c) Assume that $\operatorname{deg} Q \geq 1$ and $\operatorname{deg} P \geq 1$.

(c.1) The unique irreducible Darboux polynomials are $h_{1}=y+Q(x)(P(x)-1)$ and $h_{2}=y+Q(x)(P(x)+1)$ with cofactors $K_{1}=-Q^{\prime}(x)(Q(x) P(x)+1)$ and $K_{2}=-Q^{\prime}(x)(Q(x) P(x)-1)$, respectively;

(c.2) System (3) is not Liouvillian integrable.

The statements (a) and (b) can be checked directly from the definition of first integral. We will divide the proof of statement (c) of Theorem 2 into different sections. In section 3 we will prove Theorem 2(c.1), while the proof of Theorem 2(c.2) will be given in section 4.

Note that the main result in statement (c.1) is the uniqueness of $h_{1}$ and $h_{2}$ as irreducible Darboux polynomials because their existence follows from [18]. We remark that $\exp \left(x^{j}\right)$ are exponential factors for any generalized Liénard polynomial differential system (1).

\section{AuXiLiary nOtions AND Results}

The following result is well-known. For a proof see, for instance, Proposition 8.4 in $[5]$.

Lemma 3. Assume $f \in \mathbb{C}[x, y]$ and let $f=f_{1}^{n_{1}} \cdots f_{r}^{n_{r}}$ be its factorization into irreducible factors over $\mathbb{C}[x, y]$. Then for a polynomial differential system (1), $f=0$ is an invariant algebraic curve with cofactor $K_{f}$ if and only if $f_{i}=0$ is an invariant algebraic curve for each $i=1, \ldots, r$ with cofactor $K_{f_{i}}$. Moreover $K_{f}=n_{1} K_{f_{1}}+$ $\cdots+n_{r} K_{f_{r}}$.

Proposition 4. The following statements hold.

(a) If $E=\exp (u / v)$ is an exponential factor for the polynomial differential system (3) and $v$ is not a constant polynomial, then $v=0$ is an invariant algebraic curve.

(b) Eventually $E=\exp (u)$ can be exponential factors coming from the multiplicity of the invariant straight line at infinity.

For a geometric meaning of exponential factors and a proof of Proposition 4 see [3]. The existence of exponential factors $\exp (u / v)$ is due to the fact that the multiplicity of the invariant algebraic curve $v=0$ is larger than 1 , for more details see again [3].

The following result given in [3] characterizes the algebraic multiplicity of an invariant algebraic curve using the number of exponential factors of system (3) associated with the invariant algebraic curve.

Proposition 5. Given an irreducible invariant algebraic curve $v=0$ of degree $k$ of system (3), it has algebraic multiplicity $\ell$ if and only if the vector field associated 
to system (3) has $\ell-1$ exponential factors $\exp \left(u_{i} / v^{i}\right)$, where $u_{i}$ is a polynomial of degree at most $i k$ and $\left(u_{i}, v\right)=1$ for $i=1, \ldots, \ell-1$.

In view of Proposition 5 if we prove that $e^{u / v}$ is not an exponential factor with $\operatorname{deg} u \leq \operatorname{deg} v$, there are no exponential factors associated to the invariant algebraic curve $v=0$.

We say that a $C^{1}$ function $V=V(x, y)$ is an integrating factor if it satisfies

$$
X V=-\operatorname{div} X V,
$$

where div stands for the divergence of the vector field $X$.

In 1992 Singer [16] proved that a polynomial differential system has a Liouvillian first integral, if and only if it has an integrating factor of the form

$$
\exp \left(\int U_{1}(x, y) d x+\int U_{2}(x, y) d y\right),
$$

where $U_{1}$ and $U_{2}$ are rational functions which verify $\partial U_{1} / \partial y=\partial U_{2} / \partial x$. In 1999 Christopher [2] improved the results of Singer showing that there are integrating factors of the form

$$
\exp (u / v) \prod_{i=1}^{k} f_{i}^{\lambda_{i}}
$$

where $u, v$ and $f_{i}$ are polynomials and $\lambda_{i} \in \mathbb{C}$. From the Darboux theory of integrability (see $[5,8,16])$ we have the following result.

Theorem 6. The polynomial differential system (3) has a Liouvillian first integral if and only if system (3) has an integrating factor of the form (4), or equivalently there exist $p$ invariant algebraic curves $f_{i}=0$ with cofactors $K_{i}$ for $i=1, \ldots, p, q$ exponential factors $E_{j}=\exp \left(u_{j} / v_{j}\right)$ with cofactors $L_{j}$ for $j=1, \ldots, q$ and $\lambda_{j}, \mu_{j} \in \mathbb{C}$ not all zero such that

$$
\sum_{i=1}^{p} \lambda_{i} K_{i}+\sum_{j=1}^{q} \mu_{j} L_{j}=- \text { divergence of }(3)=f(x) .
$$

\section{Proof of Theorem 2(c.1)}

The proof of Theorem 2(c.1) will be a direct consequence of some auxiliary results.

Proposition 7. Let $h=h(x, y)$ be a Darboux polynomial of system (3) with cofactor $K \neq 0$. Then $K=K(x)$.

Proof. From the fact that system (3) has degree equal to $\operatorname{deg} g=2 \operatorname{deg} f+1=$ $m+1 \geq 3$, and that $K$ is a polynomial of degree at most $m$ we write $K$ as

$$
K(x, y)=\sum_{j=0}^{m} K_{j}(x) y^{j},
$$

where $K_{j}(x)$ has degree at most $m-j$. By assumptions $h$ satisfies

$$
y \frac{\partial h}{\partial x}-(g(x)+f(x) y) \frac{\partial h}{\partial y}=h \sum_{j=0}^{m} K_{j}(x) y^{j},
$$


where $f$ and $g$ were given in (3). We write $h(x, y)=\sum_{j=0}^{l} h_{j}(x) y^{j}$. Without loss of generality we can assume that $h_{l}(x) \neq 0$. Computing the coefficient of $y^{l+m}$ in (6) we get

$$
0=h_{l}(x) K_{m}(x) \text { that is } K_{m}(x)=0 .
$$

Therefore repeating this argument for $y^{l+m-1}, \ldots, y^{l+2}$, we get that $K_{j}(x)=0$ for $j=2, \ldots, m-1$. Hence $K(x)=K_{0}(x)+K_{1}(x) y$. Computing the coefficient of $y^{l+1}$ in (6) we get $h_{l}^{\prime}(x)=h_{l}(x) K_{1}(x)$ that is

$$
h_{l}(x)=C \exp \left(\int K_{1}(x) d x\right), \quad C \in \mathbb{C} .
$$

Since $h_{l}(x)$ must be a polynomial in $x$ we have that $K_{1}(x)=0$. This completes the proof of the proposition.

Proposition 8. The unique irreducible Darboux polynomials of system (3) with non-zero cofactor are $h_{1}=y+Q(x)(P(x)-1)$ and $h_{2}=y+Q(x)(P(x)+1)$ with cofactors, respectively, $K_{1}=-Q^{\prime}(x)(Q(x) P(x)+1)$ and $K_{2}=-Q^{\prime}(x)(Q(x) P(x)-$ $1)$.

Proof. By direct computations we get that $h_{1}$ and $h_{2}$ are irreducible Darboux polynomials of system (3).

Now we shall prove that these are the only irreducible Darboux polynomials of system (3). Let $h=h(x, y)$ be another irreducible Darboux polynomial of system (3) with cofactor $K$. In view of Proposition 7 we have that $K=K(x)$. Then,

$$
y \frac{\partial h}{\partial x}-(g(x)+f(x) y) \frac{\partial h}{\partial y}=K(x) h,
$$

with $f$ and $g$ as in (3).

Now we introduce the variables $(X, Y)$ with

$$
X=x \quad \text { and } \quad Y=h_{1}=y+Q(x)(P(x)-1) .
$$

Then in these variables system (3) becomes

$$
X^{\prime}=Y-Q(X)(P(X)-1), \quad Y^{\prime}=-Q^{\prime}(X)(Q(X) P(X)+1) Y .
$$

Let $h=\bar{h}(X, Y)$. Then, if we denote by $\tilde{h}=\tilde{h}(X)$ the restriction of $\bar{h}$ to $Y=0$ we get that $\tilde{h} \neq 0$ (otherwise $h$ would not be irreducible). Note that $\tilde{h}$ is a Darboux polynomial of system (8) restricted to $Y=0$, that is,

$$
-Q(X)(P(X)-1) \frac{d \tilde{h}}{d X}=K(X) \tilde{h}
$$

where $K(X)$ is the cofactor of $\tilde{h}$, equal to the cofactor of $h$.

Solving this linear differential equation we deduce that

$$
\tilde{h}=C \exp \left(-\int \frac{K(X)}{Q(X)(P(X)-1)} d X\right), \quad C \in \mathbb{C} \backslash\{0\} .
$$

Let $r(X)=-Q(X)(P(X)-1)$. Without loss of generality we can assume that $K$ and $r$ are coprime, otherwise we divide by their common factor. We claim that

$$
\operatorname{deg} K<\operatorname{deg} r .
$$


We proceed by contradiction. Assume that and consider the Euclidean division of $K$ and $r$. We have

$$
K(X)=s(X) r(X)+\psi(X)
$$

where $\psi(X)$ cannot be zero taking into account that $K$ and $r$ are coprime and $\operatorname{deg} \psi<\operatorname{deg} r$. Hence equation (12) becomes

$$
\frac{K(X)}{r(X)}=s(X)+\frac{\psi(X)}{r(X)} .
$$

Integrating this equation and taking into account (10) we have that

$$
\tilde{h}(X)=C \exp (\tilde{s}(X)) \exp \left(\int \frac{\psi(X)}{r(X)} d X\right), \quad C \in \backslash\{0\}
$$

where $\tilde{s}^{\prime}(X)=s(X)$. Therefore, the first factor in (14) cannot cancel with the second factor of (14), and this gives a contradiction with the fact that $\tilde{h}(X)$ is a polynomial. Hence, we conclude that $\operatorname{deg} K<\operatorname{deg} r$, which proves (11).

We say that the polynomial $r(X)$ is square-free if $r(X)=\prod_{l=1}^{k}\left(X-\alpha_{l}\right)$ with $\alpha_{l} \neq \alpha_{l}$ for $l, j=1, \ldots, k$ and $l \neq j$. We claim that

$$
\text { the polynomial } r \text { must be square free. }
$$

We again proceed by contradiction. Using an affine transformation of the form $X \mapsto X+\alpha$ with $\alpha \in \mathbb{C}$ if necessary, we can assume that $X$ is a factor of the polynomial $r$ with multiplicity $\mu>1$. Then we write it as $r(X)=X^{\mu} s(X)$ with $s(0) \neq 0$. We know that $K(0) \neq 0$ since $K$ and $r$ are coprime. Now we develop $K(X) / r(X)$ in simple fractions of $X$, that is

$$
\frac{K(X)}{r(X)}=\frac{c_{\mu}}{X^{\mu}}+\frac{c_{\mu-1}}{X^{\mu-1}}+\cdots+\frac{c_{1}}{X}+\frac{\alpha_{1}(X)}{s(X)},
$$

where $\alpha_{1}(X)$ is a polynomial with $\operatorname{deg} \alpha_{1}<\operatorname{deg} s$ and $c_{i} \in \mathbb{C}$, for $i=1,2, \ldots, \mu$. Equating both expressions we get that $c_{\mu}=K(0) / s(0) \neq 0$. Therefore equation (10) becomes

$$
\tilde{h}(X)=C \exp \left(\frac{c_{\mu}}{1-\mu} \cdot \frac{1}{X^{\mu-1}}\right) \exp \left[\int\left(\frac{c_{\mu-1}}{X^{\mu-1}}+\cdots+\frac{c_{1}}{\mu}+\frac{\alpha_{1}(X)}{s(X)}\right) d X\right],
$$

where $C \in \mathbb{C} \backslash\{0\}$. The first exponential cannot be simplified with any part of the second exponential. Thus, we get a contradiction with the fact that $\tilde{h}$ must be a polynomial. Therefore we conclude that $r$ must be square-free and (15) is proved.

Hence we have

$$
\frac{K(X)}{r(X)}=\frac{\gamma_{1}}{X-\alpha_{1}}+\cdots+\frac{\gamma_{k}}{X-\alpha_{k}} .
$$

Integrating (10) we get

$$
\tilde{h}(X)=C\left(X-\alpha_{1}\right)^{\gamma_{1}}\left(X-\alpha_{2}\right)^{\gamma_{2}} \cdots\left(X-\alpha_{k}\right)^{\gamma_{k}}, \quad C \in \mathbb{C} \backslash\{0\} .
$$

Since $\tilde{h}$ must be a polynomial we must have that $\gamma_{i} \in \mathbb{N} \cup\{0\}$ for $i=1, \ldots, k$.

Now we introduce the variables $(X, Y)$ with

$$
X=x \quad \text { and } \quad Y=h_{2}=y+Q(x)(P(x)+1) .
$$

Then in these variables system (3) becomes

$$
X^{\prime}=Y-Q(X)(P(X)+1), \quad Y^{\prime}=-Q^{\prime}(X)(Q(X) P(X)-1) Y .
$$


Let $h=\hat{h}(X, Y)$. Then, if we denote by $h^{*}=h^{*}(X)$ the restriction of $\hat{h}$ to $Y=0$ we get that $h^{*} \neq 0$ (otherwise $h$ would not be irreducible). Here $h^{*}$ is a Darboux polynomial of system (18) restricted to $Y=0$, that is

$$
-Q(X)(P(X)+1) \frac{d h^{*}}{d X}=K(X) h^{*} .
$$

Solving this linear differential equation we deduce that

$$
h^{*}=C_{1} \exp \left(-\int \frac{K(X)}{Q(X)(P(X)+1)} d X\right), \quad C_{1} \in \mathbb{C} \backslash\{0\} .
$$

Proceeding as we did for $\tilde{h}$, if we denote by $r^{*}(X)=-Q(X)(P(X)+1)$, then we must have that $r^{*}$ is square free and that

$$
\frac{K(X)}{r^{*}(X)}=\frac{\delta_{1}}{X-\beta_{1}}+\cdots+\frac{\delta_{\ell}}{X-\beta_{\ell}} .
$$

Integrating (19) we get

$$
h^{*}(X)=C_{1}\left(X-\beta_{1}\right)^{\delta_{1}}\left(X-\beta_{2}\right)^{\delta_{2}} \cdots\left(X-\beta_{\ell}\right)^{\delta_{\ell}}, \quad C_{1} \in \mathbb{C} \backslash\{0\} .
$$

Since $h^{*}$ must be a polynomial we must have that $\beta_{i} \in \mathbb{N} \cup\{0\}$ for $i=1, \ldots, \ell$.

Note that if we denote by $h=h(x, y)$ a Darboux polynomial of system (3) with cofactor $K=K(x)$ then

$$
h=\tilde{h}+(y+Q(x)(P(x)-1)) h_{1}, \quad h=h^{*}+(y+Q(x)(P(x)+1)) h_{2},
$$

for some polynomials $h_{1}, h_{2} \in \mathbb{C}[x, y]$. Moreover, from (16) we obtain

$$
K(x)=-\frac{\tilde{h}^{\prime}(x)}{\tilde{h}(x)} Q(x)(P(x)-1),
$$

where the prime denotes derivative with respect to $x$, and from (20) we get

$$
K(x)=-\frac{h^{*^{\prime}}(x)}{h^{*}(x)} Q(x)(P(x)+1) .
$$

Hence

which yields

$$
\frac{\tilde{h}^{\prime}(x)}{\tilde{h}(x)}(P(x)-1)=\frac{h^{*^{\prime}}(x)}{h^{*}(x)}(P(x)+1),
$$

That is

$$
\left(\frac{\tilde{h}^{\prime}(x)}{\tilde{h}(x)}-\frac{h^{*^{\prime}}(x)}{h^{*}(x)}\right) P(x)=\frac{\tilde{h}^{\prime}(x)}{\tilde{h}(x)}+\frac{h^{*^{\prime}}(x)}{h^{*}(x)} .
$$

$$
P(x)=\frac{\tilde{h}^{\prime}(x) h^{*}(x)+\tilde{h}(x) h^{*^{\prime}}(x)}{\tilde{h}^{\prime}(x) h^{*}(x)-\tilde{h}(x) h^{*^{\prime}}(x)}=1+2 \frac{\tilde{h}(x) h^{*^{\prime}}(x)}{\tilde{h}^{\prime}(x) h^{*}(x)-\tilde{h}(x) h^{*^{\prime}}(x)}=1-\frac{2}{1-u(x)},
$$

where

$$
u(x)=\frac{h^{*}(x)}{h^{*^{\prime}}(x)} \frac{\tilde{h}^{\prime}(x)}{\tilde{h}(x)} .
$$

The rational function $u(x)$ cannot be a constant, because the polynomial $P(x)$ has degree at least one. Clearly from (21) the function $u(x)$ cannot be neither a polynomial, nor a quotient of two polynomials. This is, we have a contradiction. This concludes the proof of the proposition. 
Proposition 9. System (3) has no polynomial first integrals.

Proof. We introduce the variables $(X, Y)$ as in (7) and we get system (8). Let $h=\bar{h}(X, Y)$ be a polynomial first integral. Then, if we denote by $\tilde{h}=\tilde{h}(X)$ the restriction of $\bar{h}$ to $Y=0$, then $\tilde{h}$ satisfies (9) with $K(X)=0$, i.e.

$$
-Q(X)(P(X)-1) \frac{d \tilde{h}}{d X}=0 . \quad \text { Then } \quad \tilde{h}(X)=\tilde{c} \in \mathbb{C} .
$$

Since we can assume without loss of generality that $h$ has no constant terms, we have $\tilde{c}=0$ and thus $\tilde{h}=0$.

Now, introducing the variables $(X, Y)$ as in (17) we get system (18). Then if we denote by $h^{*}=h^{*}(X)$ the restriction of $\hat{h}$ to $Y=0$, then $h^{*}$ satisfies (20) with $K(X)=0$, i.e.

$$
-Q(X)(P(X)+1) \frac{d h^{*}}{d X}=0 . \quad \text { Then } \quad h^{*}(X)=c^{*} \in \mathbb{C} .
$$

Since we can assume without loss of generality that $h$ has no constant terms, we have $c^{*}=0$ and thus $h^{*}=0$.

In short any polynomial first integral $h$ can be written as

$$
h=(y+Q(x)(P(x)-1)) g_{1}, \quad h=(y+Q(x)(P(x)+1)) g_{2},
$$

for some polynomials $g_{1}, g_{2} \in \mathbb{C}[x, y]$. Hence,

$$
h=\left[(y+Q(x) P(x))^{2}-Q(x)^{2}\right] g_{3},
$$

for some $g_{3} \in \mathbb{C}[x, y]$ that satisfies

$y \frac{\partial g_{3}}{\partial x}-\left(Q(x) Q^{\prime}(x)\left(Q(x)^{2} P(x)^{2}-1\right)+\left(3 Q(x) Q^{\prime}(x) P(x)+Q(x)^{2} P^{\prime}(x)\right) y\right) \frac{\partial g_{3}}{\partial y}=K g_{3}$,

with $K=2 Q^{\prime}(x) Q(x) P(x)$. In other words $g_{3}$ must be a Darboux polynomial of system (3) with cofactor $K=2 Q^{\prime}(x) Q(x) P(x)$. In view of Proposition 8 and Lemma 3 we must have

$$
m_{1} K_{1}(x)+m_{2} K_{2}(x)=2 Q^{\prime}(x) Q(x) P(x), \quad m_{1}, m_{2} \in \mathbb{N} \cup\{0\},
$$

were $K_{1}(x)=-Q^{\prime}(x)(Q(x) P(x)+1)$ and $K_{2}(x)=-Q^{\prime}(x)(Q(x) P(x)-1)$. This is not possible because $m_{1}$ and $m_{2}$ must be positive integers, and this contradiction completes the proof of the proposition.

Proof of Theorem 2(c.1). The proof of Theorem 2(c.1) follows directly from Propositions 8 and 9 .

\section{Proof of Theorem 2(c.2)}

We divide the proof of Theorem 2 in different steps.

Lemma 10. System (3) has no exponential factors of the form $\exp (u / h)$ with $u$ and $h$ coprime and $\operatorname{deg} u<\operatorname{deg} h$, being $h$ one of the two irreducible Darboux polynomials of Proposition 8. 
Proof. Let $h_{1}=y+Q(x)(P(x)-1)$ and $E=\exp \left(u / h_{1}\right)$ with $u$ and $h_{1}$ being coprime. Clearly, after simplifying by $\exp \left(u / h_{1}\right)$, we get that $u$ satisfies

$$
\begin{aligned}
& y \frac{\partial u}{\partial x}-\left(Q(x) Q^{\prime}(x)\left(Q(x)^{2} P(x)^{2}-1\right)+\left(3 Q(x) Q^{\prime}(x) P(x)+Q(x)^{2} P^{\prime}(x)\right) y\right) \frac{\partial u}{\partial y} \\
& \quad+Q^{\prime}(x)(Q(x) P(x)+1) u=L(x, y) h_{1},
\end{aligned}
$$

where $L$ is a polynomial of degree at most $m$. We introduce the change of variables of (7) and equation (22) becomes

$$
\begin{aligned}
& (Y-Q(X)(P(X)-1)) \frac{\partial \bar{u}}{\partial X}-Q^{\prime}(X)(Q(X) P(X)+1) Y \frac{\partial \bar{u}}{\partial Y} \\
& \quad+Q^{\prime}(X)(Q(X) P(X)+1) \bar{u}=\bar{L} Y,
\end{aligned}
$$

where $\bar{u}=\bar{u}(X, Y)=u(x, y)$ and $\bar{L}=\bar{L}(X, Y)=L(x, y)$. If we denote by $\tilde{u}$ the restriction of $\bar{u}$ to $Y=0$ we have that $\tilde{u} \neq 0$ (otherwise $\bar{u}$ would be divisible by $Y$ ). Evaluating (23) on $Y=0$ we conclude that

$$
-Q(X)(P(X)-1) \frac{d \tilde{u}}{d X}+Q^{\prime}(X)(Q(X) P(X)+1) \tilde{u}=0 .
$$

Therefore $\tilde{u}$ must be a polynomial that satisfies $(9)$ with $K(X)=-Q^{\prime}(X)(Q(X) P(X)+$ $1)$. Note that proceeding as in the proof of Proposition 8 we get that deg $K(X)$ must be less than the degree of $Q(X)(P(X)-1)$, which is not the case. Hence, system (3) has no exponential factors of the form $\exp \left(u / h_{1}\right)$ with $u$ and $h_{1}$ being coprime.

Let $h_{2}=y+Q(x)(P(x)+1)$ and $E=\exp \left(u / h_{2}\right)$ with $u$ and $h_{2}$ being coprime. After simplifying by $u / h_{2}$, we get that $u$ satisfies

$$
\begin{aligned}
& y \frac{\partial u}{\partial x}-\left(Q(x) Q^{\prime}(x)\left(Q(x)^{2} P(x)^{2}-1\right)+\left(3 Q(x) Q^{\prime}(x) P(x)+Q(x)^{2} P^{\prime}(x)\right) y\right) \frac{\partial u}{\partial y} \\
& \quad+Q^{\prime}(x)(Q(x) P(x)-1) u=L(x, y) h_{2},
\end{aligned}
$$

where $L$ is a polynomial of degree at most $m$. We introduce the change of variables of (17) and equation (24) becomes

$$
\begin{aligned}
& (Y-Q(X)(P(X)+1)) \frac{\partial \bar{u}}{\partial X}-Q^{\prime}(X)(Q(X) P(X)-1) Y \frac{\partial \bar{u}}{\partial Y} \\
& \quad+Q^{\prime}(X)(Q(X) P(X)-1) \bar{u}=\bar{L} Y,
\end{aligned}
$$

where $\bar{u}=\bar{u}(X, Y)=u(x, y)$ and $\bar{L}=\bar{L}(X, Y)=L(x, y)$. If we denote by $\tilde{u}$ the restriction of $\bar{u}$ to $Y=0$ we have that $\tilde{u} \neq 0$ (otherwise $\bar{u}$ would be divisible by $Y$ ). Evaluating (25) on $Y=0$ we conclude that

$$
-Q(X)(P(X)+1) \frac{d \tilde{u}}{d X}+Q^{\prime}(X)(Q(X) P(X)-1) \tilde{u}=0 .
$$

Note that proceeding as in the proof of Proposition 8 we get that the degree of $\left(Q^{\prime}(X)(Q(X) P(X)-1)\right)$ must be less than the degree of $Q(X)(P(X)+1)$, which is not the case. Hence, system (3) has no exponential factors of the form $\exp \left(u / h_{2}\right)$ with $u$ and $h_{2}$ being coprime.

In view of Proposition 5 and Lemma 10, system (3) has no exponential factors of the form $E=\exp \left(u / h_{j}^{n}\right)$ with $u \in \mathbb{C}[x, y]$ coprime with $h_{j}$ for $j=1,2$ and $n \geq 1$. 
Then the unique possible exponential factors of system (3) are of the form $e^{u}$ with $u \in \mathbb{C}[x, y]$.

Lemma 11. If system (3) has a Liouvillian first integral, then it has an integrating factor of the form $\exp (u(x, y)) h_{1}^{\lambda_{1}} h_{2}^{\lambda_{2}}$ where $u \in \mathbb{C}[x, y], \lambda_{1}, \lambda_{2} \in \mathbb{C}$ and $h_{1}$ and $h_{2}$ are the Darboux polynomials of Theorem 2 (c.1). Moreover the cofactor of the exponential factor $\exp (u(x, y))$ is a polynomial $L=L(x)$.

Proof. Let $L(x, y)$ be the cofactor of $\exp (u(x, y))$. In order that system (3) has a Liouvillian first integral, by Theorems 6, Theorem 2 (c.1) and Lemma 10 we must have

$$
\begin{aligned}
& -\lambda_{1} Q^{\prime}(x)(Q(x) P(x)+1)-\lambda_{2} Q^{\prime}(x)(Q(x) P(x)-1)+L(x, y)=f(x)= \\
& =3 Q(x) Q^{\prime}(x) P(x)+Q(x)^{2} P^{\prime}(x) .
\end{aligned}
$$

We expand $L$ in power series in the variable $y$ as $L(x, y)=\sum_{j=0}^{m} L_{j}(x) y^{j}$. Computing the coefficients of $y^{j}$ with $j>0$ in $(26)$ we get that $L_{j}(x)=0$ for $j=1, \ldots, n$ and thus $L=L_{0}(x)$. This concludes the proof.

Since we are looking for Liouvillian first integrals of system (3), in view of Lemma 11 , we can restrict to study the exponential factors with cofactor $L=L(x)$.

Proposition 12. System (3) has no exponential factors of the form $\exp (u)$ where $u \in \mathbb{C}[x, y]$ with cofactor $L=L(x)$.

Proof. Let $E=\exp (u)$ with $u \in \mathbb{C}[x, y] \backslash \mathbb{C}$ and let $L=L(x)=\sum_{k=0}^{m} \beta_{k} x^{k}$ be the cofactor associated to $E$ with $\beta_{k} \in \mathbb{C}$. We write $u=\sum_{j=0}^{r} u_{j}(x) y^{j}$. Without loss of generality we can assume that $u_{r}(x) \neq 0$. By the definition of exponential factor (2) we have

$$
y \frac{\partial u}{\partial x}-(g(x)+f(x) y) \frac{\partial u}{\partial y}=\sum_{k=0}^{m} \beta_{k} x^{k},
$$

with $f$ and $g$ as in (3). Then

$$
\begin{aligned}
& \sum_{j=1}^{r} u_{j}^{\prime}(x) y^{j+1}-Q(x) Q^{\prime}(x)\left(Q(x)^{2} P(x)^{2}-1\right) \sum_{j=1}^{r} j u_{j}(x) y^{j-1} \\
& -Q(x)\left(3 Q^{\prime}(x) P(x)+Q(x) P^{\prime}(x)\right) \sum_{j=1}^{r} j u_{j}(x) y^{j}=\sum_{k=0}^{m} \beta_{k} x^{k} .
\end{aligned}
$$

We write

$$
Q(x)=a_{q} x^{q}+\text { l.o.t. } \text { and } P(x)=b_{p} x^{p}+\text { l.o.t },
$$

where l.o.t denotes the lower order terms in $x$.

Now we consider two cases.

Case 1: $r \geq 2$. Computing in (28) the coefficient of $y^{r+1}$ we get that $u_{r}^{\prime}(x)=0$, that is, without loss of generality we can take $u_{r}(x)=1$. Now we claim that if we write

$$
u=u(x, y)=y^{r}+\sum_{j=1}^{r} u_{r-j}(x) y^{r-j},
$$


then for $j=1, \ldots, r$

$$
u_{r-j}(x)=\frac{\left(a_{q}^{2} b_{p}\right)^{j} A_{j}}{j !(2 q+p)^{j}} x^{j(2 q+p)}+\text { l.o.t. },
$$

where $A_{1}=(3 q+p) r, A_{2}=q(2 q+p) r+(3 q+p)^{2} r(r-1)$, and for $\ell \geq 2$,

$$
A_{\ell+1}=(3 q+p)(r-\ell) A_{\ell}+q \ell(2 q+p)(r-\ell-1) A_{\ell-1} .
$$

Note that in view of $(30)$ we have that $A_{\ell+1}>0$ for any $\ell=0, \ldots, r-1$.

We start the proof of the claim. For $j=1$ computing the coefficient of $y^{r}$ in (28), we get that

$$
u_{r-1}^{\prime}(x)=r Q(x)\left(3 Q^{\prime}(x) P(x)+Q(x) P^{\prime}(x)\right)=r(3 q+p) a_{q}^{2} b_{p} x^{2 q+p-1}+\text { l.o.t. }
$$

Integrating it we obtain

$$
u_{r-1}(x)=\frac{a_{q}^{2} b_{p}(3 q+p) r}{2 q+p} x^{2 q+p}+\text { l.o.t. }
$$

which coincides with (29) for $j=1$.

For $j=2$ computing the coefficient of $y^{r-1}$ in (28) we get that

$$
\begin{aligned}
u_{r-2}^{\prime}(x) & =Q(x)\left(Q^{\prime}(x)\left(Q(x)^{2} P(x)^{2}-1\right) r\right. \\
& +Q(x)\left(3 Q^{\prime}(x) P(x)+Q(x) P^{\prime}(x)\right)(r-1) u_{r-1}(x) .
\end{aligned}
$$

Now using that $u_{r-1}(x)=\frac{a_{q}^{2} b_{p}(3 q+p) r}{(2 q+p)} x^{(2 q+p)}+$ l.o.t. we obtain

$$
\begin{aligned}
u_{r-2}^{\prime}(x) & =q a_{q}^{4} b_{p}^{2} r x^{4 q+2 p-1}+(3 q+p) a_{q}^{2} b_{p}(r-1) x^{2 q+p-1} \frac{a_{q}^{2} b_{p}(3 q+p) r}{2 q+p} x^{2 q+p}+\text { l.o.t. } \\
& =q a_{q}^{4} b_{p}^{2} r x^{4 q+2 p-1}+a_{q}^{4} b_{p}^{2} \frac{(3 q+p)^{2} r(r-1)}{2 q+p} x^{4 q+2 p-1}+\text { l.o.t. } \\
& =\frac{a_{q}^{4} b_{p}^{2}}{2 q+p}\left(q(2 q+p) r+(3 q+p)^{2} r(r-1)\right) x^{4 q+2 p-1}+\text { l.o.t. } \\
& =\frac{a_{q}^{4} b_{p}^{2} A_{2}}{2 q+p} x^{4 q+2 p-1}+\text { l.o.t. }
\end{aligned}
$$

Integrating it we get

$$
u_{r-2}(x)=\frac{a_{q}^{4} b_{p}^{2} A_{2}}{2 !(2 q+p)^{2}} x^{4 q+2 p}+\text { l.o.t. }
$$

which coincides with (29) for $j=2$.

Now we assume that (29) holds for $j=0, \ldots, L$ with $L<r$ and we will prove it for $j=L+1$. Computing the terms in (28) with $y^{r-L}$ we get

$$
\begin{aligned}
u_{r-L-1}^{\prime}(x) & =Q^{\prime}(x) Q(x)^{3} P(x)^{2}(r-L+1) u_{r-L+1}(x) \\
& +Q(x)\left(3 Q^{\prime}(x) P(x)+Q(x) P^{\prime}(x)\right)(r-L) u_{r-L}(x)+\text { l.o.t. }
\end{aligned}
$$


Now using the induction hypothesis and (30) we obtain that

$$
\begin{aligned}
u_{r-L-1}^{\prime}(x) & =q a_{q}^{4} b_{p}^{2} x^{4 q+2 p-1}(r-L+1) \frac{\left(a_{q}^{2} b_{p}\right)^{L-1} A_{L-1}}{(L-1) !(2 q+p)^{L-1}} x^{(L-1)(2 q+p)} \\
& +(3 q+p) a_{q}^{2} b_{p} x^{2 q+p-1}(r-L) \frac{\left(a_{q}^{2} b_{p}\right)^{L} A_{L}}{L !(2 q+p)^{L}} x^{L(2 q+p)}+\text { l.o.t. } \\
& =\frac{\left(a_{q}^{2} b_{p}\right)^{L+1}}{L !(2 q+p)^{L}} x^{(L+1)(2 q+p)-1}\left(q L(2 q+p)(r-L+1) A_{L-1}\right. \\
& \left.+(3 q+p)(r-L) A_{L}\right)+ \text { l.o.t. } \\
& =\frac{\left(a_{q}^{2} b_{p}\right)^{L+1} A_{L+1}}{L !(2 q+p)^{L}} x^{(L+1)(2 q+p)-1}+\text { l.o.t. }
\end{aligned}
$$

Integrating the previous equation yields

$$
\begin{aligned}
u_{r-L-1}(x) & =\frac{\left(a_{q}^{2} b_{p}\right)^{L+1} A_{L+1}}{L !(2 q+p)^{L}(L+1)(2 q+p)} x^{(L+1)(2 q+p)}+\text { l.o.t. } \\
& =\frac{\left(a_{q}^{2} b_{p}\right)^{L+1} A_{L+1}}{(L+1) !(2 q+p)^{L+1}} x^{(L+1)(2 q+p)}+\text { l.o.t. },
\end{aligned}
$$

which is equation (29) with $j=L+1$. This completes the proof of the claim.

From (29) with $j=r-1$ we obtain

$$
u_{1}(x)=\frac{\left(a_{q}^{2} b_{p}\right)^{r-1} A_{r-1}}{(r-1) !(2 q+p)^{r-1}} x^{(r-1)(2 q+p)}+\text { l.o.t. }
$$

We recall that $A_{r-1}>0$. Now computing the coefficient of $y^{0}$ in (28) we get

$$
-Q(x) Q^{\prime}(x)\left(Q(x)^{2} P(x)^{2}-1\right) u_{1}(x)=\sum_{k=0}^{m} \beta_{0, k} x^{k} .
$$

Using (31) the degree of the polynomial in the left hand side of $(32)$ is $(r-1)(2 q+$ $p)+4 q+2 p-1 \geq 6 q+3 p-1$. Since the degree of the right-hand side is at most $m=4 q+2 p-2$, we have a contradiction.

Case 2: $r \leq 1$. We write $u=u(x, y)=u_{0}(x)+u_{1}(x) y$. Computing the coefficient of $y^{2}$ in (28) we get

$$
u_{1}^{\prime}(x)=0, \quad \text { and without loss of generality we can take } u_{1}(x)=1 .
$$

Furthermore the coefficient of $y$ in (28) gives

$$
u_{0}^{\prime}(x)-\left(3 Q(x) Q^{\prime}(x) P(x)+Q(x)^{2} P^{\prime}(x)\right)=0,
$$

that is

$$
u_{0}(x)=\beta^{0}+\int\left(3 Q(x) Q^{\prime}(x) P(x)+Q(x)^{2} P^{\prime}(x)\right) d x,
$$

being $\beta^{0}$ a constant.

Finally the coefficient of $y^{0}$ in (28) gives

$$
-Q(x) Q^{\prime}(x)\left(Q(x)^{2} P(x)^{2}-1\right)=\sum_{k=0}^{m} \beta_{k} x^{k} .
$$

Since $g(x)=Q(x) Q^{\prime}(x)\left(Q(x)^{2} P(x)^{2}-1\right)$ has degree $m+1$, from (34) we get a contradiction. This concludes the proof of the proposition. 
Proof of Theorem 2(c.2). The proof of Theorem 2 follows directly from Lemma 11 and Proposition 12.

\section{ACKNOWLEDGEMEnTS}

The first author is partially supported by a MINECO/ FEDER grant number MTM2008-03437, an AGAUR grant number 2009SGR 410, ICREA Academia, two FP7-PEOPLE-2012-IRSES numbers 316338 and 318999, and FEDER-UNAB10-4E378. The second author is supported by Portuguese National Funds through FCT Fundação para a Ciência e a Tecnologia within the project PTDC/MAT/117106/2010 and by CAMGSD.

\section{REFERENCES}

[1] L. Cairó, H. Giacomini and J. Llibre, Liouvillian first integrals for the planar LotkaVolterra system, Rend. Circ. Mat. Palermo 52 (2003), 389-418.

[2] C.J. Christopher, Liouvillian first integrals of second order polynomial differential equations, Electron. J. Differential Equations 1999 (1999), pp 7.

[3] C. Christopher, J. Llibre and J.V. Pereira, Multiplicity of invariant algebraic curves and Darboux integrability, Pacific J. Math., 229 (2007), 63-117.

[4] G. Darboux, Mémoire sur les équations différentielles algèbriques du premier ordre et du premier degré (Mélanges), Bull. Sci. Math. 2ème. série 2 (1878), 60-96; 123-144; 151-200.

[5] F. Dumortier, J. Llibre And J.C. Artés, Qualitative theory of planar differential systems, UniversiText, Springer-Verlag, New York, 2006.

[6] R. Iannacci, M.N. Nkashama, P. Omari and F. Zanolin, Periodic solutions of forced Liénard equations with jumping nonlinearities under nonuniform conditions, Proc. Roy. Soc. Edinburgh Sect. A 110 (1988), 183-198.

[7] S. LABRunie, On the polynomial first integrals of the $(a, b, c)$ Lotka-Volterra system, J. Math. Phys. 37 (1996), 5539-5550.

[8] J. LliBRE, Integrability of polynomial differential systems, Handbook of Differential Equations, Ordinary Differential Equations, Eds. A, Canada, P. Drabek and A. Fonda, Elsevier (2004), pp. 437-533.

[9] J. Llibre AND C. VAlls, Liouvillian first integrals for Liénard polynomial differential systems, Proc. Amer. Math. Soc. 138 (2010), 3229-3239.

[10] J. Llibre AND C. VAlLs, Liouvillian first integrals for generalized Liénard polynomial differential systems, Advanced Nonlinear Studies 13 (2013), 819-829.

[11] J. LliBRE AND C. VALLS, The generalized Liénard polynomial differential systems $x^{\prime}=y$, $y^{\prime}=-g(x)-f(x) y$ with $\operatorname{deg} g=\operatorname{deg} f+1$ are not Liouvillian integrable, preprint 2013.

[12] J. Moulin Ollagnier, Polynomial first integrals of the Lotka-Volterra system, Bull. Sci. math. 121 (1997), 463-476.

[13] J. Moulin Ollagnier, Rational integration of the Lotka-Volterra system, Bull. Sci. Math. 123 (1999), 437-466.

[14] J. Moulin OLlaGnieR, Liouvillian Integration of the Lotka-Volterra system, Qual. Theory Dyn. Syst. 2 (2001), 307-358.

[15] J. Moulin Ollagnier, Corrections and complements to: "Liouvillian integration of the LotkaVolterra system" [Qual. Theory Dyn. Syst. 2 (2001), 307-358], Qual. Theory Dyn. Syst. 5 (2004), 275-284.

[16] M. F. Singer, Liouvillian first integrals of differential systems, Trans. Amer. Math. Soc. 333 (1992), 673-688.

[17] L. Yang And X.W. Zeng, The period function of Liénard systems, Proc. Roy. Soc. Edinburgh Sect. A 143 (2013), 205-221.

[18] H. Zoladek, Alegebraic invariant curves for the Liénard quation, Trans. Amer. Math. Soc. 350 (1998), 1681-1701. 
1 Departament de Matemàtiques, Universitat Autònoma de Barcelona, 08193 Bellaterra, Barcelona, Catalonia, Spain

E-mail address: jllibre@mat.uab.cat

2 Departamento de Matemática, Instituto Superior Técnico, Universidade de Lisboa, Av. Rovisco Pais 1049-001, Lisboa, Portugal

E-mail address: cvalls@math.ist.utl.pt 